\title{
Frecuencia de ideación suicida en una población de anestesiólogos
}

\author{
Ideation of suicidal in a population of anesthesiologists \\ Samuel da Rosa Sousa', Claudio Luciano Franck², Kristian Madeira ${ }^{3}$, Pedro Gabriel Ambrosio³, \\ Sarah Galatto Cancillier ${ }^{4}$
}

\begin{abstract}
Introduction: Due to the absence of precise data regarding the occupational and mental health of Anesthesiologists (MA), in this study. Objectives: We sought to identify the frequency of suicidal ideation in Anesthesiologists of a Brazilian state. The goals was to verify the frequency of suicidal ideation and common variables in the Anesthesiologists of the selected population, in order to make a literary contribution and generate knowledge that may be useful, through mental and occupational health strategies. Method: An individual, observational, cross-sectional study was conducted, characterized as a research, with a quantitative approach, among anesthesiologists, by means of an electronic questionnaire. Results: 266 questionnaires were applied between September 2018 and November 2018, of which all those who did not answer the questionnaire or did so partially were excluded, as well as those who did not perform the profession in the state of Paraná, resulting in $n=233$. Discussion: It was verified that $14.59 \%$ (34) of the anesthesiologists in the sample have experienced suicidal thoughts. Suicidal ideation conceptually addresses thoughts, ideas, planning or desire to end life
\end{abstract}

\section{RESUMEN}

Introducción: Debido a la ausencia de datos precisos respecto de la salud

\section{Key words:}

Anesthesiologists, suicidal

\section{Palabras clave:}

Suicidio, anestesiología

Médico en Especialización en Anestesiología, Escola de Saúde Pública de São José dos Pinhais. PR.

Médico Anestesiólogo, Doctorado en Principios de la Cirugía en el Instituto de Pesquisas Médicas, Brasil (2017).

Doctor en Ciencias de la Salud (2015), Magíster en Educación (2009), Especialista en Educación Matemática (2004) y Licenciado en Ciencias y Matemática (2001) en la Universidade do Extremo Sul Catarinense (UNESC).

4 Matemático, Universidade do Extremo Sul Catarinense (UNESC).

Este trabajo recibió el premio al mejor resumen presentado por residentes en el Congreso Latinoamericano de Anestesia 2019.

Fecha de recepción: 24 de enero de 2020

Fecha de aceptación: 22 de febrero de 2020

\section{ORCID}

http://orcid.org/0000-0002-4957-7412

\section{Correspondencia:}

Samuel da Rosa Sousa

sr.sousa@live.com 
laboral y mental de los Médicos Anestesiólogos (MA), en este estudio, se buscó identificar la frecuencia de ideación suicida en anestesiólogos de un Estado brasileño. Objetivos: Verificar la frecuencia de ideación suicida y variables comunes en los anestesiólogos de la población seleccionada, con el fin de realizar una contribución literaria y generar conocimiento que pueda ser útil, por medio de estrategias de salud mental y laboral. Método: Se realizó un estudio individual, observacional, transversal, caracterizado como investigación, de enfoque cuantitativo, entre médicos anestesiólogos, por medio de un cuestionario electrónico. Resultados: Se aplicaron 266 cuestionarios entre septiembre de 2018 y noviembre de 2018 , de los cuales se excluyeron a todos aquellos que no respondieron el cuestionario o lo hicieron de forma parcial, así como aquellos que no desempeñaban la profesión en el Estado de Paraná, dando como resultado $n=233$. Discusión: Se verificó que el 14,59\% (34) de los médicos anestesiólogos de la muestra han experimentado pensamientos suicidas. La ideación suicida conceptualmente aborda pensamientos, ideas, planeación o deseo de terminar con la vida.

\section{Introducción}

$\mathrm{D}$ ebido a la ausencia de datos precisos respecto de la salud laboral y mental de los Médicos Anestesiólogos (MA), en este estudio, se buscó identificar la frecuencia de ideación suicida en anestesiólogos de un Estado brasileño.

\section{Material y Métodos}

La muestra se compone de un número de 233 MA. Los datos recolectados fueron analizados con ayuda del software IBM Statistical Package for the Social Sciencies (SPSS), versión 23.0. Las variables cuantitativas fueron expresadas por medio de mediana y amplitud intercuartil. Las pruebas estadísticas fueron realizadas con un nivel de significancia $p=0,05$. La distribución de los datos respecto de la normalidad fue evaluada por medio de la aplicación del test de Kolmogorov-Smirnov. La investigación de la existencia de asociación entre las variables cualitativas se realizó por medio de la aplicación de las pruebas Chicuadrado de Pearson, Razón de Verosimilitud y Exacta de Fisher, seguidas de análisis de residuo, cuando se observó significancia estadística. Las variables cuantitativas fueron expresadas por medio de mediana y amplitud intercuartil. Las pruebas estadísticas fueron realizadas con un nivel de significancia $p=0,05$. La distribución de los datos respecto de la normalidad fue evaluada por medio de la aplicación del test de Kolmogorov-Smirnov. La investigación de la existencia de asociación entre las variables cualitativas se realizó por medio de la aplicación de las pruebas Chicuadrado de Pearson, Razón de Verosimilitud y Exacta de Fisher, seguidas de análisis de residuo, cuando se observó significancia estadística.

\section{Resultados}

Los resultados encontrados respecto de la distribución de las variables estudiadas se encuentran en la Tabla 1.

Se verificó que el 14,59\% (34) de los médicos anestesiólogos han experimentado pensamientos suicidas. De estos profesionales expuestos, 44,1\% (15) $(p=0,656)$ pertenecen a la franja etaria de 30 a 39 años, $61,8 \%(20)(p=0,741)$ son de sexo masculino, $70,6 \%(24)(p=0,741)$ son casados, 50\% (17) $(p=$ $0,695)$ poseen tiempo de formación de entre 10 y 29 años, $79,4 \%(27)(p=0,529)$ refirieron como mayor título académico el de especialista en anestesiología. El $64,7 \%(22)(p=0,812)$ de estos se concentran en la capital, siendo el régimen autónomo 39\% (23) ( $p$ $=0,660$ ) el principal tipo de vínculo laboral y el convenio o particular $52,2 \%(24)(p=0,254)$ el principal tipo de institución en la que se desempeñan. La calidad de la estructura de trabajo fue relatada como buena el $50 \%(17)(p=0,211)$ de las veces. Fue posible evaluar también que, en relación con la carga horaria semanal de trabajo, el $82,2 \%(28)(p=0,077)$ trabajan de 40 a 79 horas, 32,4\% (11) $(p=0,516)$ poseen una remuneración salarial de entre 20 a 29 mil reales, $79,4 \%(27)(p=0,530)$ realizan guardias nocturnas, $23,5 \%(8)(p=0,366)$ no se sienten reali- 


\section{Tabla 1. Distribución de la muestra de los anestesiólogos de Paraná en relación con las variables estudiadas, 2018} n (\%)

¿Ha experimentado algún pensamiento suicida?

Franja etaria

$<30$ años

De 30 a 39 años

De 40 a 49 años

De 50 a 59 años

60 años o más

Sexo

Masculino

Femenino

Estado civil

Soltero

Casado

Divorciado

Viudo

Tiempo de formación

Menos de 5 años a 9 años

De 10 a 29 años

30 años o más

Mayor título académico obtenido

Superior en anestesiología

Especialista en anestesiología

Maestría

Doctorado

Posdoctorado

Región donde ejercen la profesión

Capital

Otras regiones

Tipo de vínculo laboral

CLT (Contrato de Trabajo)

Servidor público

Autónomo

Por concurso público

Persona jurídica

Tipo de institución donde se desempeñan

SUS (sistema público de salud)

Particular o convenio

Mixto

\section{Sí}

$n=34$

$1(2,9)$

$15(44,1)$

No

$\mathrm{n}=199$

Valor-p

$9(4,5)$

$0,656 \neq$

$90(45,2)$

$50(25,1)$

$33(16,6)$

$17(8,5)$

$1(2,9)$

$21(61,8)$

$13(38,2)$

$116(58,3)$

$0,704 \dagger$

$83(41,7)$

$5(14,7)$

$37(18,6)$

$0,741 \neq$

$24(70,6)$

$142(71,4)$

$5(14,7)$

$19(9,5)$

$0(0,0)$

$1(0,5)$

$14(41,1)$

$76(38,2)$

$0,695 \neq$

17 (50)

$93(46,7)$

$3(8,8)$

$30(15,1)$

$4(11,8)$

$18(9,0)$

0,529‡

$27(79,4)$

$3(8,8)$

$158(79,4)$

$14(7,0)$

$6(3,0)$

$3(1,5)$

$0(0,0)$

$22(64,7)$

$12(35,4)$

$139(69,8)$

$60(30)$

$3(5,1)$

$9(15,2)$

$23(39,0)$

$2(3,4)$

$22(37,3)$

$34(9,7)$

$0,223+$

$25(7,2)$

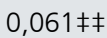

$142(40,7)$

$0,660+$

$27(7,7)$

$0,270 \neq \ddagger$

$121(34,7)$

$0,666+$

$12(26,1)$

$78(27,6)$

$0,666+$

$24(52,2)$

$120(42,4)$

$0,254+$

$10(21,7)$

$85(30,0)$

$0,145+$ 


\begin{tabular}{|c|c|c|c|}
\hline \multicolumn{4}{|c|}{ Calidad de la estructura de trabajo } \\
\hline Mala & $0(0,0)$ & $11(5,5)$ & $0,211 \neq$ \\
\hline Regular & $10(29,4)$ & $45(22,6)$ & \\
\hline Buena & $17(50,0)$ & $111(55,8)$ & \\
\hline Excelente & $7(20,6)$ & $32(16,1)$ & \\
\hline \multicolumn{4}{|c|}{ Carga horaria semanal de trabajo } \\
\hline Menos de 20 horas & $0(0,0)$ & $5(2,5)$ & $0,077 \ddagger$ \\
\hline De 20 a 39 horas & $3(8,8)$ & $32(16,1)$ & \\
\hline De 40 a 59 horas & $14(41,1)$ & $104(52,2)$ & \\
\hline De 60 a 79 horas & $14(41,1)$ & $42(21,1)$ & \\
\hline 80 horas o más & $3(8,8)$ & $16(8,0)$ & \\
\hline \multicolumn{4}{|l|}{ Remuneración salarial } \\
\hline Menos de 10 mil reales & $0(0,0)$ & $4(2,0)$ & $0,516 \neq$ \\
\hline De 10 a 19 mil reales & $5(14,7)$ & $34(17,1)$ & \\
\hline De 20 a 29 mil reales & $11(32,4)$ & $68(34,2)$ & \\
\hline De 30 a 39 mil reales & $7(20,6)$ & $55(27,6)$ & \\
\hline De 40 a 49 mil reales & $7(20,6)$ & $22(11,1)$ & \\
\hline Más de 50 mil reales & $4(11,8)$ & $16(8,0)$ & \\
\hline \multicolumn{4}{|l|}{ Realizan guardia nocturna } \\
\hline Sí & $27(79,4)$ & $148(74,4$ & $0,530+$ \\
\hline No & $7(20,6)$ & $51(25,6)$ & \\
\hline \multicolumn{4}{|c|}{ Se sienten profesionalmente satisfechos } \\
\hline Sí & $26(76,5)$ & $165(82,9)$ & $0,366+$ \\
\hline No & $8(23,5)$ & $34(17,1)$ & \\
\hline \multicolumn{4}{|c|}{ Harían nuevamente la especialización en anestesiología } \\
\hline Sí & $24(70,6)$ & $157(78,9)$ & $0,282+$ \\
\hline No & $10(29,4)$ & $42(21,1)$ & \\
\hline \multicolumn{4}{|c|}{ Se consideran actualizados en el trabajo } \\
\hline Sí & $21(61,8)$ & $156(78,4) b$ & $0,036+$ \\
\hline No & $13(38,2) b$ & $43(21,6)$ & \\
\hline \multicolumn{4}{|l|}{ Frecuencia con la que estudian } \\
\hline Estudian con regularidad & $17(50,0)$ & $103(51,8)$ & $0,850+$ \\
\hline No estudian con regularidad & $17(50,0)$ & $96(48,2)$ & \\
\hline \multicolumn{4}{|c|}{ Última participación en congresos } \\
\hline Menos de 1 año & $22(64,7)$ & $121(63,0)$ & $0,858 \neq$ \\
\hline Entre 1 y 2 años & $5(14,7)$ & $37(19,3)$ & \\
\hline Entre 2 y 3 años & $3(8,8)$ & $11(5,7)$ & \\
\hline Entre 3 y 4 años & $2(5,9)$ & $7(3,6)$ & \\
\hline Entre 4 y 5 años & $2(5,9)$ & $16(8,3)$ & \\
\hline \multicolumn{4}{|c|}{ Realizan contribución social o privada } \\
\hline Sí & $32(94,1)$ & $184(92,5)$ & $0,999 \neq \neq$ \\
\hline
\end{tabular}




\begin{tabular}{|c|c|c|c|}
\hline No & $2(5,9)$ & $15(7,5)$ & \\
\hline \multicolumn{4}{|c|}{ Franja etaria en la que imaginan jubilarse } \\
\hline De 40 a 49 años & $1(3,7)$ & $2(1,2)$ & \multirow[t]{3}{*}{$0,282 \neq$} \\
\hline De 50 a 59 años & $5(18,5)$ & $51(31,5)$ & \\
\hline 60 años o más & $21(77,8)$ & $109(67,3)$ & \\
\hline \multicolumn{4}{|c|}{ Toman vacaciones completas por 30 días } \\
\hline Sí & $9(26,5)$ & $47(23,6)$ & \multirow[t]{2}{*}{$0,719+$} \\
\hline No & $25(73,5)$ & $152(76,4)$ & \\
\hline \multicolumn{4}{|l|}{ Consideran que duermen bien } \\
\hline Sí & $12(35,2)$ & $91(45,7)$ & \multirow[t]{2}{*}{$0,258+$} \\
\hline No & $22(64,7)$ & $108(54,3)$ & \\
\hline \multicolumn{4}{|c|}{ Se sienten habitualmente estresados } \\
\hline Sí & $31(91,2) b$ & $108(54,3)$ & \multirow[t]{2}{*}{$<0,001 \dagger$} \\
\hline No & $3(8,8)$ & $91(45,7) b$ & \\
\hline \multicolumn{4}{|c|}{ Últimamente, se han sentido deprimidos } \\
\hline Sí & $11(32,4) b$ & $25(12,6)$ & \multirow[t]{2}{*}{$0,003+$} \\
\hline No & $23(67,6)$ & $174(87,4) b$ & \\
\hline \multicolumn{4}{|c|}{ Cómo consideran la relación familiar } \\
\hline Mala & $1(2,9)$ & $3(1,5)$ & \multirow[t]{4}{*}{$0,012 \neq$} \\
\hline Regular & $10(29,4) b$ & $17(8,5)$ & \\
\hline Buena & $15(44,1)$ & $102(51,3)$ & \\
\hline Excelente & $9(23,5)$ & $77(38,7)$ & \\
\hline \multicolumn{4}{|l|}{ Usan ansiolíticos o antidepresivos } \\
\hline Sí & $16(47,1) b$ & $40(20,1)$ & \multirow[t]{2}{*}{$0,001+$} \\
\hline No & $18(52,9)$ & $159(79,9) b$ & \\
\hline \multicolumn{4}{|l|}{ Consumen bebidas alcohólicas } \\
\hline No consumen & $10(29,4)$ & $59(29,6)$ & \multirow[t]{3}{*}{$0,833 \neq$} \\
\hline 1 a 3 veces por semana & $23(67,6)$ & $130(65,4)$ & \\
\hline 4 veces por semana o más & $1(2,9)$ & $10(5,0)$ & \\
\hline \multicolumn{4}{|l|}{ Tienen el hábito de fumar } \\
\hline No & $30(88,2)$ & $186(93,5)$ & \multirow[t]{3}{*}{$0,451+$} \\
\hline Sí, menos de 1 paquete por día & $4(11,8)$ & $12(6,0)$ & \\
\hline Sí, más de 1 paquete por día & $0(0,0)$ & $1(0,5)$ & \\
\hline \multicolumn{4}{|c|}{ Practican actividad física al menos 5 veces por semana } \\
\hline Sí & $6(17,6)$ & $64(32,2)$ & \multirow[t]{2}{*}{$0,106 \neq \ddagger$} \\
\hline No & $28(82,4)$ & $135(67,8)$ & \\
\hline
\end{tabular}

††Valor obtenido por medio de la aplicación de la prueba U de Mann-Whitney; †Valores obtenidos por medio de la aplicación de la prueba Chi-cuadrado de Pearson; Valores obtenidos por medio de la aplicación de la prueba Razón de Verosimilitud.

b: Diferencias estadísticamente significativas luego del análisis de residuo.

Fuente: Datos de investigación, 2019. 
zados profesionalmente, 73,5\% (25) ( $p=0,719)$ no toman vacaciones completas por 30 días y $29,4 \%$ (10) $(p=0,282)$ refieren que no harían nuevamente la especialización en anestesiología. De los expuestos al pensamiento suicida, el $61,8 \%(21)(p=0,036)$ se consideran actualizados profesionalmente, 50\% (17) $(p=0,850)$ estudian con regularidad, 64,7\% (22) ( $p$ $=0,858$ ) participaron de algún congreso en el último año. Este grupo de la muestra refirió en el 94, $1 \%$ (32) ( $p=0,999)$ de las veces que realizan contribución social y privada, demostrando, en la mayoría de los casos, 77,8\% (21) ( $p=0,282)$ de intención de jubilarse con 60 años o más. En relación con los aspectos psicológicos y hábitos de vida, el 64,7\% (22) ( $p=0,258)$ consideran que no duermen bien, 91,2\% (31) ( $p<$ $0,001)$ se sienten habitualmente estresados y $32,4 \%$ (11) $(p=0,003)$ se han sentido deprimidos últimamente. Para el 44,1\% (15) ( $p=0,012)$, la relación familiar fue descrita como buena. También dentro de la misma temática, se observó que, de aquellos que experimentaron pensamientos suicidas, 47,1\% (16) $(p=0,001)$ refirieron el uso de ansiolíticos o antidepresivos, 67,6\% (23) relataron consumo de bebidas alcohólicas de 1 a 3 veces por semana, 82,2\% (30) ( $p$ $=0,451)$ no fuman y $82,5 \%(28)(p=0,106)$ no practican actividad física al menos 5 veces por semana.

\section{Discusión}

Se verificó que el 14,59\% (34) de los médicos anestesiólogos de la muestra han experimentado pensamientos suicidas. La ideación suicida conceptualmente aborda pensamientos, ideas, planeación o deseo de terminar con la vida[1],[2]. Informes señalaron que el suicidio explicaría alrededor del 35\% de las muertes prematuras de los médicos en los Estados Unidos. En Australia, específicamente en la población de médicos anestesiólogos, el 13,2\% de los evaluados relataron que tuvieron pensamientos suicidas en los últimos 12 meses[4]. En Brasil, aproximadamente el $5 \%$ de los médicos presentan ideación suicida[5]. En relación con el sexo, el predominio de ideación fue del sexo masculino 61,8\% (20) ( $p=0,741)$. En Finlandia, el $22,1 \%$ de los hombres y el $25,9 \%$ de las mujeres médicas presentaban historial de ideación[6].

Se observó que el 44,1\% (15) ( $p=0,656)$ de aquellos con ideación pertenecen a la franja etaria de 30 a 39 años. Estudios señalan que, en poblaciones jóvenes, principalmente aquellos menores de 30 años, presentan mayor riesgo de ideación suicida en comparación con profesionales de mayor edad[7],[8]. En relación con el tiempo de formación, el 50\% (17)
( $p=0,695)$ poseían un tiempo de formación de entre 10 y 29 años. Generalmente, el tiempo de formación es proporcional a la edad de los médicos, reflejando que aquellos con hasta cinco años de formación tienen, como máximo, 30 años y, en esta muestra estudiada, existe una franja joven expuesta[5].

La frecuencia de profesionales casados 70,6\% (24) $(p=0,741)$ y la frecuencia de relación familiar descrita como buena 44,1\% (15) ( $p=0,012)$ en el grupo de expuestos contradice lo señalado por los estudios, según los cuales ser divorciado, soltero, estar en proceso de separación o con una relación familiar mala aumenta las chances de ideación[9].

Frecuentemente, el mayor título académico referido $79,4 \%(27)(p=0,529)$ fue el de especialista en anestesiología, estando estos profesionales concentrados en la capital $64,7 \%(22)(p=0,812)$, trabajando como autónomos 39\% (23) ( $p=0,660)$, teniendo como principal tipo de vínculo laboral convenio o particular $52,2 \%(24)(p=0,254)$ y refiriendo la calidad de la estructura de trabajo como buena en el $50 \%$ (17) $(p=0,211)$ de los casos. Desde el punto de vista psicológico, podemos afirmar que los hospitales no se encuentran entre los mejores lugares de trabajo, dado que estos profesionales ven su salud amenazada por factores de riesgo como la convivencia con enfermedades terminales, accidentes, sufrimiento, muerte, intentos de tratamiento sin éxito y responsabilidad sobre las decisiones, afectando considerablemente sus vidas, principalmente cuando esta estructura es precaria, hecho que puede reflejar la concentración de profesionales en la capital y en el sector privado que, en la mayoría de los casos, brindan mejores condiciones de trabajo[7],[10].

La carga horaria semanal de trabajo de aqueIlos con ideación suicida fue de 40 a 79 horas en el $82,2 \%(28)(p=0,077)$ de los casos. La combinación de alta carga de trabajo e involucramiento emocional son factores de riesgo para el origen del estrés y la ideación suicida, situación resaltada en un estudio en el cual el $40 \%$ de aquellos formados en anestesiología fueron considerados con alto riesgo de burnout, condición correlacionada con el estrés y el riesgo de suicidio[8],[11].

También en relación con los aspectos profesionales, la remuneración salarial del 32,4\% (11) ( $p=$ $0,516)$ oscila entre 20 a 29 mil reales, con realización de guardias nocturnas por el 79,4\% (27) $(p=0,530)$ de los profesionales afectados. A pesar de que el $61,8 \%(21)(p=0,036)$ de aquellos expuestos a la ideación suicida se consideraran actualizados profesionalmente, el $50 \%(17)(p=0,850)$ estudian con regularidad, $64,7 \%(22)$ ( $p=0,858)$ han participado 
de algún congreso en el último año, la insatisfacción profesional fue señalada por el $23,5 \%(8)(p=0,366)$ de estos profesionales, y el 29,4\% (10) $(p=0,282)$ indicaron que no harían nuevamente la especialización en Anestesiología. Los mayores factores de insatisfacción profesional están relacionados con el estrés, las guardias y la baja remuneración. Se verifica que la satisfacción profesional de los médicos ha disminuido con el correr de los años. En 1973, menos de 15\% relataba dudas sobre haber escogido la profesión correcta, mientras que actualmente alrededor de 30\% a $40 \%$ de los médicos refieren que no harían nuevamente medicina, si pudieran elegir, y se quejan con frecuencia respecto de la renta salarial y la carga de trabajo[12].

De la muestra, el 91,2\% (31) ( $p<0,001)$ refirieron sentirse habitualmente estresados, 32,4\% (11) $(p=0,003)$ últimamente se han sentido deprimidos y $47,1 \%(16)(p=0,001)$ refirieron el uso de ansiolíticos o antidepresivos. El estrés representa una dolencia que se debe a múltiples factores. De una manera aguda, puede presentar manifestaciones clínicas de fatiga, depresión, y llevar a la ideación suicida y al suicidio[13],[14]. Al compararse con datos de Australia, se verifica en ese país una prevalencia de depresión del 7,1\% entre anestesiólogos[16].

En relación con los hábitos y el estilo de vida de aquellos que han experimentado ideación suicida, el $64,7 \%(22)(p=0,258)$ consideran que no duermen bien, $73,5 \%(25)(p=0,719)$ no toman vacaciones completas por 30 días, 67,6\% (23) relataron consumo de bebidas alcohólicas de 1 a 3 veces por semana, $82,2 \%(30)$ ( $p=0,451)$ no fuman y $82,5 \%$ (28) $(p=0,106)$ no practican actividad física al menos 5 veces por semana. La mala alimentación asociada al sedentarismo, privación del sueño, el uso de cafeína y el aislamiento social parecen contribuir de forma significativa en la generación de fatiga, estrés, depresión, y propiciar el abuso de sustancias, factores que podrían conducir a una mayor chance de ideación y suicidio[17]. Un estudio mostró que el 15,3\% de los profesionales médicos abusan del alcohol, siendo la anestesiología una de las especialidades con las mayores tasas[18].

Por último, en relación con la planeación en cuanto a la finalización de las actividades profesionales y jubilación, de los expuestos a la ideación suicida, el 94,1\% (32) ( $p=0,999)$ refirió realizar contribución social y privada, demostrando, en la mayoría de los casos $77,8 \%(21)(p=0,282)$, intención de jubilarse con 60 años o más. Se observa una situación peculiar en la población estudiada, ya que, incluso con tasas elevadas de estrés y estado de ánimo deprimido, pretenden jubilarse tardíamente. La literatura muestra que el estrés elevado puede generar falta de interés en el trabajo, mala calidad, problemas jurídicos y otras condiciones que podrían llevar a una jubilación prematura y, en casos extremos, al suicidio[19],[20].

\section{Conclusiones}

La evaluación de la ideación suicida de los médicos anestesiólogos de Paraná encontró una frecuencia de $14,59 \%$ (34) y demuestra que los expuestos a la ideación suicida profesionales son, en su mayoría, hombres, casados, de entre 30 y 49 años, que no fuman, consumen alcohol 1 a 3 veces por semana, son sedentarios y están estresados. Se consideran actualizados, estudian regularmente, frecuentan congresos y poseen el título de especialista como mayor grado académico, trabajan en hospitales con buena estructura, cumplen 40 a 80 horas semanales, no toman vacaciones completas de 30 días, realizan guardias nocturnas y duermen mal.

Vislumbran jubilarse luego de los 60 años e invierten en seguridad social. La mayoría haría anestesiología nuevamente como especialización y se considera satisfecho profesionalmente.

Señalan que la ideación suicida está relacionada con relevancia estadística con el estrés ( $p<0,001)$, con la sensación de estar deprimidos $(p=0,003)$, con el uso de ansiolíticos o antidepresivos ( $p=0,001)$.

Queda claro que proporcionar material científico para la comprensión de este grave problema es fundamental para crear, en el medio médico, concientización de que la prevención del suicidio es posible. Es necesario, también, crear programas de orientación e información, estímulo a nuevos estudios y el desarrollo de políticas de salud. 


\section{Referencias}

1. Osman A, Barrios FX, Gutierrez PM, Wrangham JJ, Kopper BA, Truelove RS, Linden SC. The Positive and Negative Suicide Ideation (PANSI) Inventory: Psychometric evalua- tion with adolescent psychiatric inpatient samples. Journal of Personality Assessment, 79, 512-530 https://doi.org/10.1207/ S15327752JPA7903_07

2. Werlang BSG, Borges VR, Fensterseifer $L$. Fatores de risco ou proteção para a presença de ideação suicida na adolescência. Revista Interamericana de Psicologia, 39, 259-266.

3. Olkinuora M, Asp S, Juntunen J, Kauttu K, Strid L, Aarimaa M. Stress symptoms, burnout, and suicide thoughts in Finnish physicians. Social Psychiatry and Psychiatric Epidemiology, 25, 81-86.

4. Bright RP, Krahn L. Depression and suicide among physicians. Curr Psychiatr. 2011;10(4):16-30.

5. Massud M, Barbosa GA, Gouveia VV. Indicadores de Saúde Mental. In: Barbosa GA, Andrade EO, Carneiro MB, Gouveia VV. A saúde dos médicos no Brasil. Brasília: Conselho Federal de Medicina; 2007. p.143-58.

6. Frank E, Dingle AD. Self-reported depression and suicide attempts among U.S. women physicians. American Journal of Psychiatry, 156, 1887-1894.

7. De Oliveira GS, Chang R, Fitzgerald PC, et al. The prevalence of burnout and depression and their association with adherence to safety and practice standards: a survey of United States an- esthesiology trainees. Anesth Anal. 2013;117:182-
193. https://doi.org/10.1213/ ANE.0b013e3182917da9

8. Shanafelt TD, Balch C, Bechamps G, et al. Burnout and medical errors among american surgeons. Ann Surg. 2010;251:1001-2 https://doi.org/10.1097/ SLA.0b013e3181bfdab3

9. Bryson EO, Silverstein JH. Addiction and substance abuse in anesthesiology. Anesthesiology. 2008;109:905-917. https://doi.org/10.1097/ ALN.0b013e3181895bc1

10. Oreskovich MR, Kaups KL, Balch CM, et al. Prevalence of alcohol use disorders among American surgeons. Arch Surg. 2012;147:168-74. https://doi.org/10.1001/archsurg.2011.1481

11. Schaufeli WB, Greenglass ER. Introduction to special issues on burnout and health. Psychology and Health. 2001;16:501-510. https://doi. 1080/08870440108405523

12. Zuger A. Dissatisfaction with medical practice. New England Journal of Medicine, 350, 69-75. https://doi.org/10.1056/NEJMsr031703

13. Andrea H, Kant IJ, Beurskens AJHM, Metsemakers J FM, Van Schayck CP. Associations between fatigue attri- butions and fatigue, health, and psychosocial work characteristics: A study among employees visiting a physician with fatigue. Occupational and Enrivonmental Medicine, 60, 90-104. https://doi.org/10.1136/ oem.60.suppl_1.i99

14. Levine RE, Bryant SG. The depressed physician: A different kind of impairment. Hospital Physician, 86, 67-73.

15. National Mental Health Survey of
Doctors and Medical Students. 2013. [Citado em 2019 Jul 20]. Disponível em: https://www. beyondblue.org.au/docs/defaultsource/research-project-files/ bl1132-report-nmhdmss-fullreport_web

16. Schernhammer ES, Colditz GA Suicide rates among physicians: a quantitative and gender assessment (meta-analysis)." Am J Psychiatry. 2004; 161(12):2295302. https://doi.org/10.1176/ appi.ajp.161.12.2295

17. Birmingham P, Ward R. A highrisk suicide group: the anesthesiologist involved in litigation. Am. J. Psychiatry. 1985;42:12251228. https://doi.org/10.1176/ ajp.142.10.1225b

18. Oreskovich MR, Shanafelt T, Dyrbye $L N$, Tan $L$, Sotile $W$, Satele $D$, West CP, Sloan J, Boone S. The prevalence of substance use disorders in American physicians. Am J Addict. 2015;24(1):308. https://doi.org/10.1111/ ajad.12173

19. Bruce D, Eide KA, Smith N, et al. A prospective survey of anesthesiologist mortality, 1967-1971. Anesthesiology. 1974;41:71-74. https://doi. org/10.1097/00000542197407000-00017

20. Bruce D, Eide KA, Linde HW, Eckenhoff JE. Causes of death among anesthesiologists: a 20 year survey. Anesthesiology. 1968:29:565-569. https:// doi.org/10.1097/00000542196805000-00039

21. Alexander B, Checkoway $\mathrm{H}$, Nagahama S, Domino K. Cause specific mortality risks of anesthesiologists. Anesthesiology. 2000;93:922-930. https:// doi.org/10.1097/00000542200010000-00008 